\title{
Is sport practice a risk factor for shoulder injuries in tetraplegic individuals?
}

\author{
GIS Medina ${ }^{1}$, CLM Jesus $^{2}$, DM Ferreira ${ }^{2}$, EMB Pacheco ${ }^{2}$, GL Beraldo ${ }^{3}$, F de Franca Urquiza ${ }^{3}$ and A Cliquet ${ }^{1,4}$
}

Study design: A retrospective cohort.

Objectives: To report the incidence rates of shoulder injuries diagnosed with magnetic resonance imaging (MRI) in tetraplegic athletes and sedentary tetraplegic individuals. To evaluate whether sport practice increases the risk of shoulder injuries in tetraplegic individuals.

Setting: Campinas, Sao Paulo, Brazil.

Methods: Ten tetraplegic athletes with traumatic spinal cord injury were selected among quad rugby athletes and had both the shoulders evaluated by MRI. They were compared with 10 sedentary tetraplegic individuals who were submitted to the same radiological protocol.

Results: All athletes were male with a mean age of 32.1 years (range 25-44 years, s.d. =6.44). Time since injury ranged from 6 to 17 years, with a mean value of 9.7 years and s.d. of 3.1 years. All sedentary individuals were male with a mean age of 35.9 years (range 22-47 years, s.d. =8.36). Statistical analysis showed a protective effect of sport in the development of shoulder injuries, with a weak correlation for infraspinatus and subscapularis tendinopathy $(P=0.09$ and $P=0.08$, respectively) and muscle atrophy $(P=0.08)$. There was a strong correlation for acromioclavicular joint (ACJ) and labrum injuries $(P=0.04)$, with sedentary individuals at a higher risk for these injuries.

Conclusion: Tetraplegic athletes and sedentary individuals have a high incidence of supraspinatus tendinosis, bursitis and ACJ degeneration. Statistical analysis showed that there is a possible protective effect of sport in the development of shoulder injuries. Weak evidence was encountered for infraspinatus and subscapularis tendinopathy and muscle atrophy $(P=0.09, P=0.08$ and $P=0.08$, respectively). Strong evidence with $P=0.04$ suggests that sedentary tetraplegic individuals are at a greater risk for ACJ and labrum injuries.

Spinal Cord (2015) 53, 461-466; doi:10.1038/sc.2014.248; published online 17 March 2015

\section{INTRODUCTION}

As a part of the rehabilitation programs, medical professionals have been utilizing sport as an effective treatment for individuals who are suffering traumatic spinal cord injury (SCI) since the end of World War II. The results are encouraging, and recent data have demonstrated the positive effects of physical activity on these patients, with evidence of improvement in both physiological and psychological aspects. ${ }^{1}$ The first major objectives were to assist in the patient's recovery and try to help in re-establishing some of their independence. ${ }^{1}$ However, today high performance is being pursued.

The Paralympic Games started in 1960, in Rome, but since 1988, in Seoul, the games officially follow the summer Olympic Games. Since then, that event is gaining popularity, and an increasing number of disabled individuals are willing to take part in the adapted sports at a high level of competition. Now many SCI individuals have the option to be professional athletes and can potentially generate sufficient income from their sports activities. Competition for lucrative marketing and sponsorship opportunities has increased the pressure to deliver a higher performance. This new incentive for results might become a burden on SCI individual's health, which has already been jeopardized by their condition.

This relatively recent change in the lifestyle of SCI subjects also should raise attention of health-care professionals regarding the injuries most commonly affecting these athletes. As far as we know, there are no papers that evaluate the shoulder of tetraplegic athletes. The objective of this study is to report the incidence of shoulder injuries in tetraplegic athletes and sedentary individuals, diagnosed by magnetic resonance imaging (MRI), and to evaluate whether highperformance sport practice increases the risk of shoulder injuries in tetraplegic individuals.

\section{PATIENTS AND METHODS}

Ten athletes with chronic traumatic SCI were conveniently selected among the national wheelchair rugby team. They all participate in the same sporting activity schedule, which includes rugby practice five times a week, each session being for a duration of $3 \mathrm{~h}$. Physical preparation is performed separately, in a 1.5-h program of muscle strengthening, three times a week. All athletes have been a part of the national team in the last year, indicating their high-level

\footnotetext{
${ }^{1}$ Department of Orthopedics and Traumatology, Faculty of Medical Sciences, State University of Campinas (UNICAMP), Sao Paulo, Brazil; ${ }^{2}$ Department of Radiology and Diagnostic Imaging, Faculty of Medical Sciences, State University of Campinas (UNICAMP), Sao Paulo, Brazil; ${ }^{3}$ Faculty of Medical Sciences, State University of Campinas (UNICAMP), Sao Paulo, Brazil and ${ }^{4}$ Department of Electrical Engineering, University of São Paulo (USP), São Carlos, Brazil

Correspondence: Dr GIS Medina, Department of Orthopedic and Traumatology, Faculty of Medical Sciences, State University of Campinas (UNICAMP), R Alexander Fleming PO Box 6111, Campinas, Sao Paulo 13081-970, Brazil.

E-mail: g008792@dac.unicamp.br
}

Received 24 November 2013; revised 26 December 2014; accepted 29 December 2014; published online 17 March 2015 
status. Ten sedentary SCI individuals were selected from the Biomechanics and Locomotor-System Rehabilitation Laboratory of the University Hospital of UNICAMP (State University of Campinas). All athletes and sedentary individuals were interviewed by the same orthopedic surgeon (GISM) for the collection of the demographic data and submitted to medical anamnesis and physical examination. Demographic data are summarized in Tables 1 and 2 and Figure 1.

We did not include individuals with acute injuries or a history of previous trauma such as fractures, dislocation, sprains or any kind of infection of the shoulder girdle. The objective was to identify chronic overload injuries, and the method chosen to evaluate this was MRI. In this study, shoulder injury was defined as any alteration seen in MRI that is a possible cause of shoulder girdle pain or dysfunction.

All participants in this study signed the informed consent form before entering the study and responded to a questionnaire regarding safety issues for the MRI, such as the presence of metallic clips, pacemaker, claustrophobia and other necessary requirements to ensure the safety of the individual at the time of examination. This study followed all the recommendations for research in human beings and was approved by our institutional review board.

MRI of the shoulder was performed with a 1.5-T scanner (Philips Medical System, Amsterdam, the Netherlands) with a dedicated shoulder coil (Sense shoulder-4). Individuals were imaged in the supine position with the arm in neutral rotation at their side. Both the shoulders of all the participants were scanned according to the following protocol:

- Axial images were obtained perpendicular to the humeral diaphysis, scanning from the top of the acromioclavicular joint up to the inferior margin of the glenohumeral $(\mathrm{GH})$ joint.

Table 1 Demographic data of the tetraplegic athletes

\begin{tabular}{lllccc}
\hline Patient & Age & $\begin{array}{c}\text { Level of } \\
\text { injury/A/S }\end{array}$ & $\begin{array}{c}\text { Time since } \\
\text { injury (years) }\end{array}$ & $\begin{array}{c}\text { Time in sport } \\
\text { practice (years) }\end{array}$ & $\begin{array}{c}\text { Rugby functional } \\
\text { classification }\end{array}$ \\
\hline 1 & 34 & C6-C7/A & 11 & 4 & 2.5 \\
2 & 44 & C5-C6/A & 11 & 4 & 2.0 \\
3 & 27 & C6-C7/C & 7 & 5 & 2.5 \\
4 & 27 & C6/A & 9 & 4 & 0.5 \\
5 & 25 & C7/A & 9 & 7 & 2.5 \\
6 & 39 & C5/A & 17 & 5 & 0.5 \\
7 & 28 & C5-C6/A & 8 & 3 & 0.5 \\
8 & 36 & C6-C7/A & 9 & 5 & 1.5 \\
9 & 26 & C6-C7/A & 6 & 4 & 1.5 \\
10 & 35 & C5/A & 7 & 2 & 0.5 \\
\hline
\end{tabular}

Abbreviations: AIS, ASIA (American Spinal Injury Association) Impairment Scale; C, cervical vertebrae level.

Table 2 Demographic data of the tetraplegic sedentary individuals

\begin{tabular}{lccc}
\hline Patient & Age (years) & Level of injury/AIS & Time since injury (years) \\
\hline 11 & 30 & C5/A & 8 \\
12 & 32 & C6-C7/B & 11 \\
13 & 41 & C6/A & 18 \\
14 & 47 & C7/A & 19 \\
15 & 46 & C6-C7/B & 18 \\
16 & 22 & C5-C6/C & 2 \\
17 & 37 & C5/A & 7 \\
18 & 27 & C5/A & 3 \\
19 & 34 & C6-C7/A & 13 \\
20 & 43 & C5-C6/A & 11
\end{tabular}

Abbreviations: AIS, ASIA (American Spinal Injury Association) Impairment Scale; C, cervical vertebrae level.
- Coronal images were obtained with images parallel to the supraspinatus tendon, from the anterior margin of the coracoid process to the posterior margin of the humeral head.

- Sagital images were acquired perpendicular to the supraspinatus tendon, from the external margin of the humeral head to the base of the coracoid process.

MRI protocol is summarized in Table 3.

This protocol was chosen in order to evaluate seven potential sources of shoulder injury: the $\mathrm{GH}$ and acromioclavicular joints (ACJs), subacromial/ subdeltoid bursae, labrum, rotator cuff (RC) tendons and muscle bellies and the long head of the biceps (LHBs) tendon.

Both glenohumeral and $\mathrm{AC}$ joints were evaluated for the presence of degenerative changes. We considered degenerative changes of the ACJ when one of the following signs was found: osteophyte, fibrous thickening of the capsule, joint space narrowing, subchondral irregularity, cartilage and bone edema. Glenohumeral joint was also evaluated for any signs of avascular necrosis, humeral head or glenoid focal cartilage lesions and osteoarthritis. Bursae were characterized regarding the presence or not of non-physiologic liquid within its limits and thickening of its membranes.

Labrum injuries were defined as labral detachments or paralabrum cysts. The tendons of the RC and LHB were analyzed looking for signal intensity alterations (tendinosis), as well as rupture signs and calcification deposits. Muscle belly atrophy or hypotrophy was evaluated as well. Figures 2 and 3 demonstrate some of the findings encountered on the MRI.

All examinations were carefully evaluated by two musculoskeletal radiologists (CLMJ and DMF) both of them blinded to the group each subject belonged.

We used binomial categorical variable (yes/no) to characterize each source of shoulder injury. Student's $t$-test for continuous variables was used. For categorical variables, a $2 \times 2$ contingency table was constructed and incidence rates of shoulder injuries among exposed (athletes) and non-exposed (sedentary) were calculated. The relative risk and the 95\% confidence interval were used to estimate the proportion of shoulder injury that hypothetically would occur because of physical activity.

\section{STATEMENT OF ETHICS}

All authors certify that all applicable institutional and governmental regulations concerning the ethical use of human volunteers were followed during the course of this research.

\section{RESULTS}

Both the groups, athletes and sedentary, were analyzed and no statistically significant differences were found regarding age, time since injury and the level of injury (Table 4 and Figure 1). They only differed regarding the exposure to sport practice. All athletes were

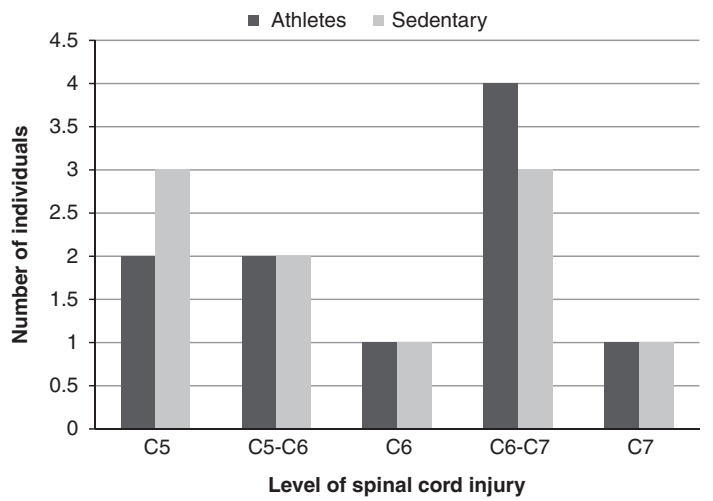

Figure 1 Distribution of the level of injury among athletes and sedentary individuals. 
male and had the same sport activity level. All sedentary individuals were male, and none of them performed any kind of physical activity.

Considering the criteria for injury that we used in this study, we identified two shoulders among the athletes and three among the sedentary that had GHJ injury. There was a high incidence of ACJ injuries in both the groups, $60 \%$ in the athletic and $90 \%$ in the sedentary group.

The RC tendons were analyzed both for the presence of tendinopathy and ruptures. The most commonly affected tendon was the supraspinatus, with 16 shoulders presenting tendinopathy among the athletes and 18 shoulders of the sedentary group. There was only one

\section{Table 3 MRI protocol}

\begin{tabular}{lccc}
\hline Sequence and plane & Section/GAP $(\mathrm{mm})$ & FOV & Other \\
\hline T2-FSE axial & $3 / 0.3$ & 16 & FAT SAT \\
PD-FSE axial & $3 / 0.3$ & 16 & \\
T2-FSE coronal & $3 / 0.3$ & 17 & FAT SAT \\
PD-FSE coronal & $3 / 0.3$ & 17 & \\
T1-SE sagital & $3.5 / 0.3$ & 17 & \\
T2-FSE sagital & $3.5 / 0.3$ & 17 & FAT SAT \\
\hline
\end{tabular}

Abbreviations: FAT SAT, fat saturation; FOV, field of view; FSE, fast spin echo; MRI, magnetic resonance imaging; $\mathrm{PD}$, proton density; $\mathrm{SE}$, spin echo. partial supraspinatus rupture in the athletic group and five in the sedentary group. None of the athletes' shoulders had infraspinatus tendinopathy or rupture, but five shoulders among the sedentary individuals had tendinopathy of this tendon. The subscapularis was affected by tendinopathy in one athletic shoulder, and in six sedentary shoulders, however, no ruptures of this tendon were identified.

The LHB tendon presented tendinopathy in two and five shoulders of the athlete and sedentary individuals, respectively. Only 1 labrum injury was identified among the 20 athletic shoulders and 8 cases of this injury in the sedentary shoulders.

A similar incidence of bursitis was present in the athletes and sedentary groups: $55 \%$ and $60 \%$, respectively. Muscle atrophy had a lower incidence rate, $5 \%$ for athletes shoulders and $30 \%$ for sedentary shoulders.

Table 5 summarizes the MRI findings among the 20 shoulders of the athletes and the 20 shoulders of the sedentary according to the criteria of injury defined in this study, as stated previously.

Statistical analysis showed a protective effect of sport regarding all potential sources of shoulder injury. However, this was statistically significant only for the ACJ and labrum injuries. Statistical results, with incidence rates for each shoulder injury among the athletes and sedentary individuals, as well as the relative risk and confidence intervals are presented in Table 6.
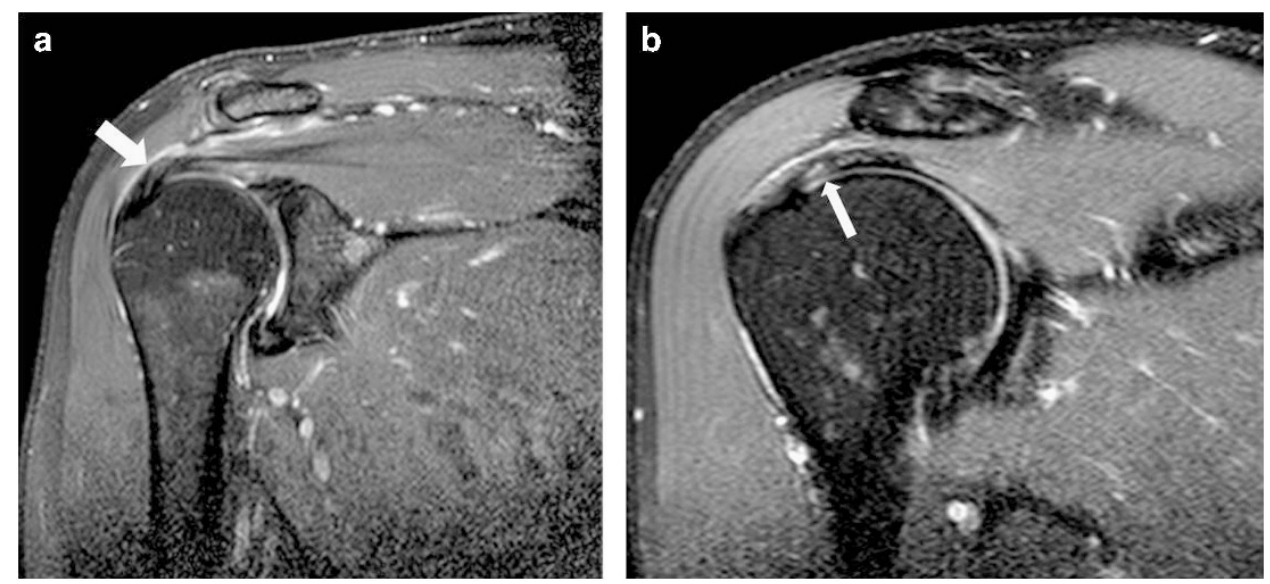

Figure 2 Coronal T2-weighted image with fat saturation. (a) Tendinopathy of the supraspinatus tendon (white arrow) and liquid in the subacromial/subdeltoid bursae; (b) Tendinopathy of the supraspinatus tendon associated with small partial intrasubstantial ruptures (white arrow).
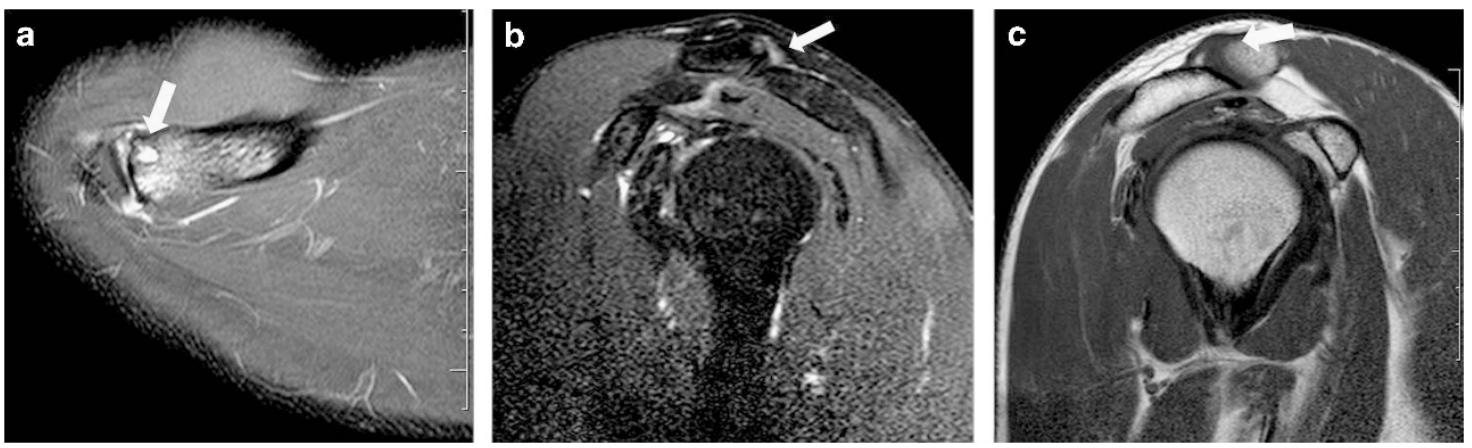

Figure 3 (a) Axial T2-weighted image with fat saturation (SPAIR): Degenerative changes in the ACJ is characterized by reduction in the articular space, articular surface irregularities, distal clavicle bone edema and subchondral cyst (white arrow). (b) Sagital T2-weighted image with fat saturation; degenerative changes in the ACJ with thickening of the capsule and mild bone edema in the distal clavicle (white arrow). (c) Sagital T1-weighted image; fibrous thickening of the ACJ capsule (white arrow). 
Table 4 Descriptive statistics for athletes and sedentary individual

\begin{tabular}{lcc}
\hline & Athletes & Sedentary \\
\hline $\begin{array}{l}\text { Age (years) } \\
\text { Range (mean) }\end{array}$ & $25-44(32.1)$ & $22-47(35.9)$ \\
s.d. & 6.44 & 8.36 \\
Time since injury (years) & & \\
Range (mean) & $6-17(9.4)$ & $2-19(11)$ \\
s.d. & 3.13 & 6.11 \\
Time in sport (years) & & - \\
Range (mean) & $2-7(4.3)$ & - \\
s.d. & 1.34 & \\
\hline
\end{tabular}

Table 5 MRI findings

\begin{tabular}{lcc}
\hline Radiographic findings & \multicolumn{2}{c}{$\begin{array}{c}\text { Number shoulders classified as } \\
\text { having an injury }\end{array}$} \\
\cline { 2 - 3 } & Athletes & Sedentary \\
\hline Glenohumeral joint & 2 & 3 \\
Acromioclavicular joint & 12 & 18 \\
Rotator cuff (tendinopathy/rupture) & & \\
$\quad$ Supraspinatus & $16 / 1$ & $18 / 5$ \\
Infraspinatus & $0 / 0$ & $5 / 0$ \\
$\quad$ Subscapularis & $1 / 0$ & $6 / 0$ \\
Long head of the biceps tendon & 2 & 5 \\
Labrum & 1 & 8 \\
Bursitis (subacromial/subdeltoidal) & 11 & 12 \\
Muscles hypotrophy & 1 & 6 \\
\hline
\end{tabular}

Abbreviation: MRI, magnetic resonance imaging.

\section{DISCUSSION}

In this study, we found a high incidence of RC pathology among both athletes and sedentary individuals, with the supraspinatus tendon being the most frequently affected. Eighty percent of the shoulders analyzed in the athlete group had supraspinatus tendinopathy and $90 \%$ of the sedentary. Previous studies reported MRI findings of the shoulder of SCI. Escobedo et al. ${ }^{2}$ found that RC pathology was evident in $71 \%$ of symptomatic paraplegic compared with $9 \%$ of asymptomatic patients. Similarly, Bayley et al. ${ }^{3}$ examined 94 patients and noted a $65 \%$ prevalence of RC tears in symptomatic shoulders in paraplegic patients. In this study, only $5 \%$ of the athlete's shoulders had a supraspinatus partial rupture and $25 \%$ for the sedentary shoulders. Alves et al. ${ }^{4}$ reported MRI findings of the shoulder of nine SCI sedentary individuals, among which five were tetraplegic. The supraspinatus tendon presented tendinosis in $60 \%$ of the tetraplegic patients. Our study reports a greater incidence of RC tendinosis in both the groups, but no statistical difference among them.

Nynomia et al. ${ }^{5}$ evaluated the shoulder of 16 sedentary SCI individuals with ultrasound, 6 of them were tetraplegic. The mean age and mean time since injury were similar to our study. They reported tendinosis in $21.4 \%$ of the $\mathrm{LHB}, 35.7 \%$ of the supraspinatus and $42.8 \%$ of the subscapularis tendon. Brose et al. ${ }^{6}$ reported $100 \%$
Table 6 Statistical results

\begin{tabular}{|c|c|c|c|c|}
\hline \multirow[t]{2}{*}{ Type of injury } & \multicolumn{2}{|c|}{ Injury incidence rate } & \multirow[t]{2}{*}{ Relative risk (CI) } & \multirow[t]{2}{*}{ P-value } \\
\hline & Athletes (\%) & Sedentary (\%) & & \\
\hline $\mathrm{GHJ}$ & 10 & 15 & $0.67(0.12-3.57)$ & 0.63 \\
\hline ACJ & 60 & 90 & $0.67(0.45-0.98)$ & $0.04^{*}$ \\
\hline SS tendinopathy & 80 & 90 & $0.89(0.68-1.15)$ & 0.38 \\
\hline SS rupture & 5 & 25 & $0.20(0.02-1.56)$ & 0.12 \\
\hline IS tendinopathy & 0 & 25 & $0.09(0.005-1.54)$ & 0.09 \\
\hline Sub tendinopathy & 5 & 30 & $0.17(0.02-1.26)$ & 0.08 \\
\hline LHB & 10 & 25 & $0.40(0.08-1.82)$ & 0.23 \\
\hline Labrum & 5 & 40 & $0.12(0.01-0.91)$ & $0.04^{*}$ \\
\hline Bursae & 55 & 60 & $0.92(0.54-1.56)$ & 0.75 \\
\hline Muscle atrophy & 5 & 30 & $0.17(0.02-1.26)$ & 0.08 \\
\hline
\end{tabular}

Abbreviations: ACJ, acromioclavicular joint; $\mathrm{Cl}$, confidence interval; $\mathrm{GHJ}$, glenohumeral joint; IS, infraspinatus; LHB, long head of the biceps; SS, supraspinatus; Sub, subscapularis.

${ }^{*} P<0.05$, statistically significant difference.

tendinosis of the supraspinatus tendon, $91.8 \%$ of the impingement syndrome and $79.6 \%$ of LHB tendinosis. However, the mean age of the participants in their study was 44.8 years and the average duration of SCI was 16.3 years, which are greater than the average of our study. They related shoulder injuries to increased age and time since injury.

In the present study, bursitis was found in 55 and $60 \%$ of the shoulders of athlete and sedentary, respectively. The tetraplegic patients evaluated by Nynomia et al. ${ }^{5}$ did not have bursitis in the US examination. Perhaps, because of the high sensitivity of MRI imaging we encountered these incidences.

Previous studies related shoulder injuries with the increase stress in the shoulder of SCI individuals, which become load-bearing joints. ${ }^{7-9}$ Our study found that sport practice has a protective effect for shoulder injuries, which in one way is opposite to this theory, as tetraplegic athletes have a greater demand on their shoulder girdle due to physical activities. We imagine that physical activity and muscle strengthening might have this protective effect because it promotes a better muscle balance in the shoulder girdle. Studies have shown that shoulder muscle imbalances may lead to the impingement syndrome and RC pathologies. ${ }^{10-12}$ This fact is corroborated by our findings that infraspinatus and subscapularis tendinopathy showed some statistical significance $(P=0.09$ and $P=0.08$, respectively), as well as muscle atrophy $(P=0.08)$. However, maybe because of the small sample size, in our study only ACJ and labrum injuries were significantly greater in sedentary SCI individuals $(P=0.04)$.

Attention should be paid to the increase incidence of degenerative changes affecting the ACJ either in the athletes and sedentary, as has been reported in the literature among non-athlete SCI individuals. ${ }^{13,14}$ It is assumed that chronic overload of the shoulder can cause degenerative osteoarticular alterations in a younger age, with authors having described alterations such as narrowing of the acromiolavicular space with marginal osteophytosis and, in some cases, clavicular osteolysis. ${ }^{15-18}$

Regardless of the symptoms, the high prevalence of shoulder injuries among SCI and its impact in their life should draw the attention of the physician to the tetraplegic sedentary individuals. When dealing with this group of individuals it is paramount to keep this in mind, in order to assess the health issues. It is important to diagnose the tendon pathology early in the disease process, to implement protective measures and therapy to avoid tearing of the $\mathrm{RC}$, as these injuries have a detrimental effect in the life of the SCI 
individual. Up to the present moment there is no consensus regarding the best treatment for RC tears in SCI individuals. Some authors report good results, but others still question surgical repair for these cases. $^{3,19}$ Therefore, the best option is to prevent these tendon ruptures.

We report here a protective effect of sport in shoulder injuries for tetraplegic individuals, although not statistically significant in all aspects studied, probably due to sample size. This may draw the attention for the need of physical activity in SCI individuals to prevent shoulder injuries.

The source of shoulder pain in SCI individuals may arise from the capsule (contracture or adhesive capsulitis) tendons (tendinosis or ruptures), GH ligaments (joint instability), bone and underlying bursae (subacromial impingement, bursitis and osteonecrosis) and joints (ACJ, GH, osteoarthiritis and condrolysis). ${ }^{8,9}$ Clinical tests are targeted to try to identify the source of pain and many of the standard clinical tests used in able-bodied individuals for investigation of shoulder pain require active movements. This may be impaired or even absent in some tetraplegic patients because of the motor deficit affecting the upper limbs. Therefore, traditional tests to identify the possible source of pain in the shoulder of these individuals are quite difficult to perform. For persons with impaired sensibility and motor function, there is no study to determine the sensitivity, specificity nor the accuracy of the standard tests in predicting shoulder pathologies. It is reasonable to think that in these cases complementary examinations may have a more important role than in able-bodied individuals. Further studies regarding the use of traditional clinical orthopedic tests in disabled individuals are needed.

MRI is a good option as a complementary examination because it is the gold standard for the evaluation of soft tissue. However, this examination cannot be used for patients with claustrophobia nor for patients with contraindications due to metallic implants and electronic devices (pacemakers, clips). It also has the disadvantage of time, cost and accessibility. On the other hand, ultrasound is cheaper, more available, less time consuming and lack contraindications. However, it is operator dependent and does not allow visualization of intraarticular structures such as the labrum, capsule and cartilage and has limited ability to evaluate intraosseous abnormalities. ${ }^{20-22}$ Therefore, we chose MRI because it enables the acquisition of images in multiple planes within one single examination, offers good anatomical visualization and all structures of the joint may be analyzed (bone, cartilage, tendons, muscle, joints). Given the fact that clinical tests are difficult to perform in SCI individuals, a complementary examination, which identifies all structures from the shoulder, is beneficial.

Limitations of this study include the small sample size and the convenience sampling method, but this is common when dealing with a specific population such as SCI individuals and even greater because of the athletic profile. We did not establish exposure levels, and it would be interesting to study the effect of different types of physical activity and different intensities in the development of shoulder injuries. A descriptive study does not allow casual inferences to be made. Therefore, cohort prospective studies or case-control studies are needed to address this issue. Strengths are the homogeneity of our population, representing the wheelchair rugby athletes and the use of the gold standard complementary examination (MRI). Descriptive studies are important to understand the characteristics of a particular group of population, and it often precedes explanatory research.

\section{CONCLUSION}

We report in this study the incidence rates of shoulder injuries diagnosed by MRI in tetraplegic athletes and sedentary individuals.
They present a high incidence of supraspinatus tendinosis, bursitis and ACJ degeneration. Statistical analysis showed that there is a possible protective effect of sport in the development of shoulder injuries. Weak evidence was encountered for infraspinatus and subscapularis tendinopathy and muscle atrophy $(P=0.09, P=0.08$ and $P=0.08$, respectively). Strong evidence with $P=0.04$ suggests that sedentary tetraplegic individuals are at a greater risk for ACJ and labrum injuries.

Further studies regarding physical examination specifically designed for disabled individuals are needed. Until then, it seems that complementary examinations are important in the evaluation of shoulder injuries among SCI individuals, to detect the diseases and elaborate a treatment protocol for shoulder injuries.

\section{DATA ARCHIVING}

There were no data to deposit.

\section{CONFLICT OF INTEREST}

The authors declare no conflict of interest.

\section{ACKNOWLEDGEMENTS}

This study was supported by the State of Sao Paulo Foundation for Research FAPESP.

1 Machida M, Irwin B, Feltz D. Resilience in competitive athletes with spinal cord injury: the role of sport participation. Qual Health Res 2013; 23: 1054-1065.

2 Escobedo E, Hunter JC, Hollister MC, Patten RM, Goldstein B. MR imaging of rotator cuff tears in individuals with paraplegia. AJR Am J Roentgenol 1997; 168: 919-923.

3 Bayley JC, Cochran TP, Sledge CB. The weight bearing shoulder: the impingement syndrome in paraplegics. J Bone Joint Surg Am 1987; 69: 676-678.

4 Alves AP, Terrabuio AA, Pimenta CJ, Medina GIS, Rimkus CM, Cliquet A. Clinical assessment and magnetic resonance imaging of the shoulder of patients with spinal cord injury. Acta Ortp Bras 2012; 20: 291-296.

5 Ninomyia AF, Jesus CLM, Auletta LL, Rimkus CM, Ferreira DM, Filho AZ et al. Shoulders of patients with spinal cord injuries submitted to rehabilitation program - a clinical and ultrasound-based assessment. Acta Ortop Bras 2007; 15: 109-113.

6 Brose SW, Boninger ML, Fullerton B, McCann T, Collinger JL, Impink BG et al. Shoulder ultrasound abnormalities, physical examination findings, and pain in manual wheelchair users with spinal cord injury. Arch Phys Med Rehabil 2008; 89: 2086-2093.

7 Silfverskiold J, Waters RL. Shoulder pain and functional disability in spinal cord injury patients. Clin Orthop Related Res 1991; 272: 141-145.

8 Campbell CC, Koris MJ. Etiologies of shoulder pain in cervical spinal cord injury. Clin Orthop Related Res 1996; 322: 140-145.

9 Hastings J, Goldstein B. Paraplegia and the shoulder. Phys Med Rehabil Clin N Am 2004; 15: 699-718.

10 Jobe FW, Kvitne RS, Giangarra CE. Shoulder pain in the overhand or throwing athlete. The relationship of anterior instability and rotator cuff impingement. Orthop Rev 1989; 18: 963-975.

11 Labriola JE, Lee TQ, Debski RE, McMahon PJ. Stability and instability of the glenohumeral joint: the role of shoulder muscles. J Shoulder Elbow Surg 2005; 14: 32S-38S.

12 Burnham RS, May L, Nelson E, Steadward R, Reid DC, May L. Shoulder pain in wheelchair athletes. The role of muscle imbalance. Am J Sports Med 1993; 21: 238-242.

13 Medina GIS, Nascimento FB, Rimkus CM, Zoppi FA, Cliquet A. Clinical and radiographic evaluation of the shoulder of spinal cord injured patients undergoing rehabilitation program. Spinal Cord 2011; 49: 1055-1061.

14 Eriks-Hoogland I, Engisch R, Brinkhof MWG, Drongelen SV. Acromioclavicular joint arthrosis in persons with spinal cord injury and able-bodied persons. Spinal Cord 2012; 51: 59-63.

15 Kivimäki J, Ahoniemi E. Ultrasonographic finding in shoulders of able-bodied, paraplegic and tetraplegic subjects. Spinal Cord 2008; 46: 50-52.

16 Lal S. Premature degenerative shoulder changes in spinal cord injury patients. Spinal Cord 1998; 36: 186-189.

17 Roach NA, Schweitzer ME. Does osteolysis of the distal clavicle occur following spinal cord injury? Skeletal Radiol 1997; 26: 16-19.

18 Boninger ML, Towers JD, Cooper RA, Dicianno BE, Munin MC. Shoulder imaging abnormalities in individuals with paraplegia. J Rehabil Res Dev 2001; 38: 401-408. 
19 Goldstein B, Young J, Escobedo EM. Rotator cuff repairs in individuals with paraplegia Am J Phys Med Rehabil 1997; 76: 316-322.

20 de Jesus JO, Parker L, Frangos AJ, Nazarian LN. Accuracy of MRI, MR arthrography, and ultrasound in the diagnosis of rotator cuff tears: a meta-analysis. AJR Am J Roent genol 2009; 192: 1701-17re07.
21 Erickson SJ. High-resolution imaging of the musculoskeletal system. Radiology 1997; 205: 593-618.

22 Middleton WD, Payne WT, Teefey SA, Hil-debolt CF, Rubin DA, Yamaguchi K. Sonography and MRI of the shoulder: comparison of patient satisfaction. AJR Am J Roentgenol 2004; 183: 1449-1452. 